\title{
BRAOYKININ-STIMULATED CHANGES IN INOSITOL PHOSPHATE MASS \\ IN RENAL PAPILLARY COLLECTING TUBULE CELLS
}

\author{
James A. Shayman* and Maureen T. Kirkwood \\ Department of Internal Medicine, \\ Veterans Administration Medical Center, and \\ University of Michigan Medical Center, \\ 3914 Taubman Center, \\ Ann Arbor, MI 48109-0364
}

Received April 28, 1937

The effect of bradykinin on changes in the chemical levels of myo-inositol polyphosphates in renal papillary collecting tubules was investigated.

Myo-inositol phosphate mass was determined by means of an enzymatic,

fluorometric assay. Bradykinin induced increases in myo-inositol mono-, bis-, and trisphosphate which were both time and concentration dependent. Furthermore, the magnitude of the chemical levels of myo-inositol monophosphate formed were unlikely to be accounted for solely by the formation and degradation of myo-inositol trisphosphate. These observations are consistent with the concomitant hydrolys is of phosphatidylinositol and phosphatidylinositol bisphosphate. This study also confirms, in freshly isolated renal tubules, observations regarding bradykinin-induced phosphatidylinositol bisphosphate hydrolysis made previously in radiolabeled cultures.

(a) 1987 Academic Press, Inc.

Renal papillary collecting tubule cells comprise the most distal portion of the mammalian nephron and, therefore, play an important role in defining the final composition of urine. However, the detailed biological responsiveness of this tissue remains poorly understood. Previous studies

\footnotetext{
* To whom correspondence may be addressed.

Abbreviations used: IP, myo-inositol monophosphate; IP, myoinositol biphosphate; $I P_{3} \frac{\text { myo-inositol triphosphate; IP }}{2}$ myo-inositol tetrakisphosphate; Quin 2-[[2-bis(carboxyl)methyl]amino]-5methylphenoxy]methyl] 6-methoxy-8-[bis(carboxyl)methyl]amino]quinalone.
} 
have documented a close association between bradykinin binding, myo-inositol trisphosphate labeling, calcium mobilization, and prostaglandin $E_{2}$ formation $(1,2)$. In these studies myo- $\left[2-{ }^{3} \mathrm{H}\right]$ inositol labeled cultures demonstrated increased activity in $I P_{1}, I P_{2}$, and $I P_{3}$ in the presence of bradykinin which occurred in a time and concentration-dependent manner. In a subsequent study, myo-inositol 1,2 cyclic monophosphate was also shown to increase in a similar manner and over a time course consistent with phosphatidylinositol hydrolysis (3). These studies, as do others of a similar design, assume when tissues are labeled to isotopic equilibrium that changes in radioactivity represent true changes in mass. This assumption has not been tested since to do so requires minimally the ability to measure inositol phosphate mass. A further observation in these studies was that $\left[{ }^{3} \mathrm{H}\right] \mathrm{P}_{3}$ formation appeared to precede $\left[{ }^{3} \mathrm{H}\right] \mathrm{IP} \mathrm{P}_{1},\left[{ }^{3} \mathrm{H}\right] \mathrm{IP} \mathrm{P}_{2}$, and $\left[{ }^{3} \mathrm{H}\right]$ inositol 1,2 cyclic monophosphate formation. It could not be ascertained, however, whether the increased labeling of $I P_{1}$ and $I P_{2}$ represented degradation of $I_{3}$ or was the result of the phosphodiesteratic hydrolysis of both phosphatidylinositol and phosphatidylinositol bisphosphate. Finally, because these cells were cultured for 3 to 5 days prior to investigation and because they were depleted of myo-inositol to promote the incorporation of radiolabel, it was unclear whether the kinetics of inositol phosphate formation reflected the true in vivo responsiveness.

To address these concerns, a recently developed assay for the determination of myo-inositol phosphate mass (4) was utilized to measure the hormonal responsiveness of freshly isolated rabbit renal papillary collecting tubule cells.

\section{MATERIALS AND METHODS}

Renal papillary collecting tubule cells were harvested from male New Zealand white rabbits as previously described (1). In brief, animals were anesthetized with pentobarbital, the kidneys were harvested, and the renal papillae were resected. Tissue from three animals was pooled and digested in Krebs buffer containing collagenase $\left(1.0 \mathrm{mg}\right.$ percent) at $37^{\circ} \mathrm{C}$ for 2 hours. Thereafter, the digestate was filtered and exposed to hypotonic buffer 
(100 mosm) for 3 minutes. Tubular fragments were pelleted and resuspended in oxygenated Krebs buffer.

$45 \mu l$ aliquots of tubular suspension, representing 150 to $400 \mu \mathrm{g}$ cell protein, were then placed in $1.5 \mathrm{ml}$ microfuge tubes. $5 \mu 1$ of buffer or of bradykinin (10-6M for time course determinations) in buffer was added to the side of the microfuge tube and gently mixed with the tubular suspension at the initiation of the incubation period. To terminate the incubation, $200 \mu 1$ of ice-cold trichloroacetic acid (1 M) was added, and the precipitated protein was pelleted by centrifugation. The supernatant was removed for inositol phosphate determination as described below. The cellular protein was resuspended in $200 \mu 1$ of $0.62 \mathrm{~N} \mathrm{NaOH}$ and assayed with the fluorescamine reagent $(5)$.

The determination of myo-inositol phosphate mass is based on the enzymatic measurement of myo-inositol as described by MacGregor and Matschinsky (6) as recently described (4). Because the application of their method to the measurement of inositol phosphates has yet to be published, it is described in detail below. The trichloroacetic acid from the supernatants was extracted by the addition of $1 \mathrm{ml}$ of water-saturated diethyl ether and removal of the upper phase. This was repeated five times or until the pH of the aqueous phase was greater than 5. The residual ether was removed by heating the sample to $65^{\circ} \mathrm{C}$ for $20 \mathrm{~min}$. The sample was applied to $0.75 \times 12 \mathrm{~cm}$ columns of Dowex AG 1 - $x 8$ formate anion exchange resin (bed volume $1 \mathrm{ml}$ ). Myo-inositol and the inositol phosphates were serially eluted with water $(16 \mathrm{ml}) ; 0.025 \mathrm{M}$ ammonium formate $(8 \mathrm{ml}) ; 0 . ? \mathrm{M}$ ammonium formate, $0.1 \mathrm{M}$ formic acid $(8 \mathrm{ml}) ; 0.4 \mathrm{M}$ ammonium formate, $0.1 \mathrm{M}$ formic acid $(8 \mathrm{ml})$; and $0.75 \mathrm{M}$ ammonium formate, $0.1 \mathrm{M}$ formic acid $(8 \mathrm{ml})$. These buffers eluted respectively myo-inositol, glycero-phosphatidyl inositol, $I P_{1}, I P_{2}$, and $I P_{3}$.

Pooled elutions containing either IP $1, I P_{2}$ or $I P_{3}$ from the separated samples were desalted and neutralized by dilution with $50 \mathrm{ml}$ of water and by addition to a $0.75 \times 12 \mathrm{~cm}$ column containing Dowex AG $1-\times 8$, chloride form (bed volume $2 \mathrm{ml}$ ); this was followed by the addition of $25 \mathrm{ml}$ of water. The respective inositol phosphates were eluted with $1.5 \mathrm{M} \mathrm{LiCl}(8 \mathrm{ml})$. Samples were brought to dryness in a vacuum centrifuge (Speed $\mathrm{Vac}$, Savant Instruments). Trapped water was removed by resuspension of the lithium salts in $4 \mathrm{ml}$ of anhydrous ethanol with reevaporation. The dried pellet, containing LiCl and the lithium salt of the inositol phosphate was resuspended in $8 \mathrm{ml}$ of absolute ethanol. The remaining salt was pelleted by centrifugation and the supernatant removed. This latter step was repeated until only a trace of pellet remained, usually 3 to 4 times.

Samples were reconstituted in $100 \mu \mathrm{l}$ of Tris buffer $(50 \mathrm{mM}, \mathrm{pH} \mathrm{9.0})$ and dephosphorylated with $50 \mathrm{U} / \mathrm{ml}$ of bovine intestinal alkaline phosphatase at $37^{\circ} \mathrm{C}$ for 2 hours. These conditions have been demonstrated to

dephosphorylate greater than 95 percent of the total inositol phosphates. The enzyme was inactivated by heating the samples for 3 minutes in a boiling water bath.

The liberated myo-inositol was chemically assayed by the protocol of MacGregor and Matschinsky (6). The following modifications were made. The assay was adapted to assay between 1 and 10 pmoles of free myo-inositol. $\mathrm{NAD}^{+}$was excluded from the first malate oxidation step since sufficient $\mathrm{NAD}^{+}$was found to remain following myo-inositol oxidation. Only $7 \mathrm{mM}$ oxaloacetate was used in the myo-inositol oxidation step. Assays were

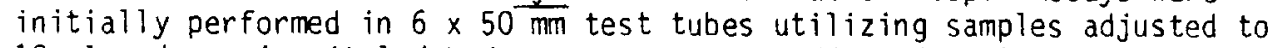
$10 \mu \mathrm{l}$ and myo-inositol dehydrogenase reagent adjusted to $2 \mu 1$. Residual oxaloacetate was destroyed with $0.5 \mu \mathrm{l}$ of $\mathrm{H}_{2} \mathrm{O}_{2}$, and residual $\mathrm{NAD}^{+}$was eliminated by the addition of $5 \mu \mathrm{l}$ of $0.35 \mathrm{~N} \mathrm{NaOH}$. The first malate oxidation was performed with $10 \mu l$ of reagent. Amplification was performed by cycling 
$5 \mathrm{ul}$ of the incubation mixture following the protocol of Kato et al. (7). $100 \mu 1$ of cycling reagent was utilized. Enzymes were adjusted to cycle 1000 times. Fluorescence was measured in a Farrand ratio fluorometer following the addition of $1 \mathrm{ml}$ of malate indicator reagent.

The recovery of the inositol phosphates varied between 75 and 90 percent and was assessed by the addition of radiolabeled standards following the addition of trichloroacetic acid. Samples were assayed in triplicate and tissue blanks in duplicate. Several blanks were determined throughout the course of each assay including duplicate tissue blanks (exluding myo-inositol dehydrogenase) for each sample, a reagent blank (excluding sample and myo-inositol dehydrogenase), a cycling blank, and a standard blank. With the exception of the tissue blanks, these were usually less than 10 percent of the fluorescence observed for 10 pmoles of myo-inositol standard and always less than 30 percent of this fluorescence. Unknown levels of myo-inositol were determined on aliquots of dephosphorylated samples varying between 1 to $10 \mu 1$ and diluted to $10 \mathrm{\mu l}$ with water by comparison to known standards measured in triplicate. Samples which did not fall with in the standard curve were repeated following adjustments in sample size.

All materials were obtained from Sigma Chemical Co. (St. Louis, MO) with the exception of malate dehydrogenase which was obtained from Boerhinger-Mannheim (Indianapolis, IN) and Dowex resins which were supplied by Bior ad (Richmond, CA).

\section{RESULTS AND DISCUSSION}

Under the incubation and assay conditions employed, inositol phosphate mass in renal papillary collecting tubule cells increased rapidly following exposure to bradykinin. Basal levels of $I P_{1}, I P_{2}$, and $I P_{3}$ were 891,83 and 124 pmoles/mg protein; these rose to maximal levels of 1953,245 and 319 pmoles/mg protein respectively (Table I). Of note is the later time at

TABLE I. TIME DEPENDENT MYO-INOSITOL PHOSPHATE FORMATION

\begin{tabular}{|c|c|c|c|c|c|c|c|c|}
\hline \multirow{2}{*}{$\begin{array}{l}\text { TIME } \\
\text { (sec) }\end{array}$} & \multicolumn{3}{|c|}{ CONTROL } & \multicolumn{5}{|c|}{ BRADYK IN IN } \\
\hline & & 10 & 300 & & 10 & 30 & 60 & 300 \\
\hline${ }^{1} P_{1}$ & 891 & \pm 236 & $793 \pm 140$ & 989 & \pm 345 & $1273 \pm 424$ & $1226 \pm 425$ & $1953 \pm 683^{*}$ \\
\hline $\mathrm{IP}_{2}$ & 83.0 & \pm 16.4 & $90.4 \pm 12.9$ & 107 & \pm 49.5 & $181 \pm 41.0 * \star$ & $111 \pm 22.0$ & $245 \pm 78.0^{\star \star}$ \\
\hline $\mathrm{IP}_{3}$ & 124 & \pm 23.0 & $135 \pm 13.4$ & 142 & \pm 14.1 & $191 \pm 17.6^{\star *}$ & $319 \pm 42 \cdot 0^{* *}$ & $244 \pm 84.5^{\star}$ \\
\hline
\end{tabular}

Myo-inositol phosphate formation in response to bradykinin $\left(10^{-7} \mathrm{M}\right)$. Papillary collecting tubule cells were incubated as described in the Methods section. Data represents the mean \pm SEM from 5-6 determinations. $\star p<0.05 ; \star \star p<0.01$ by unpaired $t$ analysis. 
which the inositol phosphates attained maximal concentrations as opposed to the previous radiolabeling studies. In those studies $I P_{3}$ peaked by 15 to 30 seconds and approached baseline by 1 to 2 minutes. On the one hand, this may reflect the less efficient mixing of effector and tissue in the present study. On the other hand, the inositol phosphates measured by radiolabeling in the prior study may not have been at equal specific activity in spite of the fact that the tissue was at isotopic equilibrium.

The level of $\mathrm{IP}_{3}$ is comparable to that reported previously in platelets utilizing gas chromatographic analys is of myp-inositol (8) and to other tissues as reported recently by Meek (9). In this latter study the recovery of myo-inositol trisphosphate was found to be highly variable depending on the tissue studied and extraction conditions employed. Preliminary observations in cortical brain slices utilizing the current methodology have confirmed this observed variability (Shayman, JA and Agranoff, BW, unpublished observations).

of potential significance is the observation that the total mass of IP and $I P_{2}$ accumulating within the first 5 minutes of kinin stimulation is in excess of that which could reasonably be accounted for by the formation and subsequent degradation of $\mathrm{IP}_{3}$. In the present study the integrated mass of $I P_{1}$ and $I P_{2}$ produced was 5.5 times that of $I P_{3}$. Thus, unless the rates of production and metabolism of $I_{3}$ were significantly greater than the dephosphorylation of $I P_{1}$ and $I P_{2}$, it is likely that there exists concomitant hydrolysis of both phosphatidylinositol and phosphatidylinositol bisphosphate. This observation supports those of wilson et al. (10) in thrombin-stimulated platelets. In that study measurements of the specific activity of the inositol containing phospholipids were consistent with the concurrent hydrolys is of both phosphatidylinositol and phosphatidylinositol bisphosphate. However, a more rigorous demonstration of concurrent phosphatidylinositol and phosphatidylinositol bisphosphate hydrolys is in both platelets and renal epithelia will require measurements of true specific activity in myo- $\left[2-{ }^{3} \mathrm{H}\right]$ inositol labeled tissues. This should now be possible with the existing methodology. 


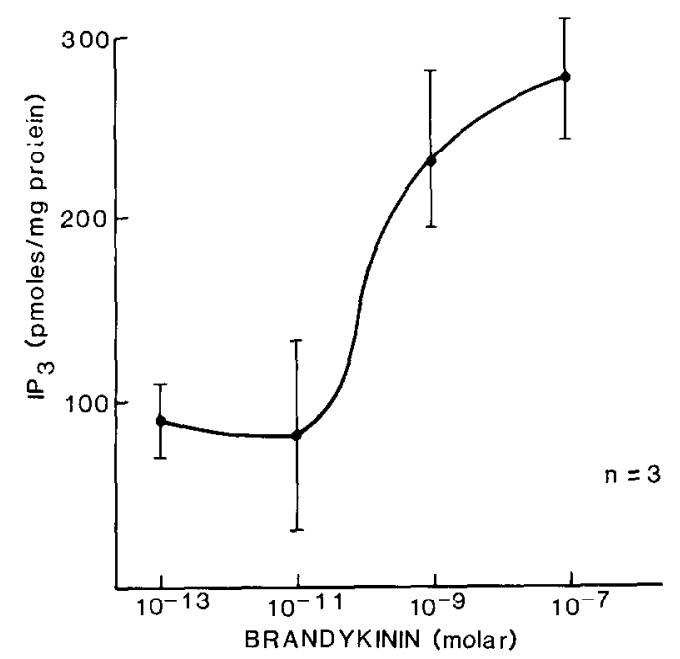

Figure 1. Concentration dependent rise in myo-inositol trisphosphate in response to $10^{-7} \mathrm{M}$ bradyk inin. Freshly isolated renal tubules were stimulated as described in Methods. Points represent the mean of three experiments in duplicate \pm SEM.

Figure 1 displays the concentration dependence of $\mathrm{IP}_{3}$ formation in response to bradykinin. The effective concentration yielding 50 percent of the maximal formation of $I P_{3}$ was $10^{-10} \mathrm{M}$. This is consistent with previous observations of both prostaglandin $E_{2}$ formation and intracellular calcium mobilization utilizing Quin $2(1,2)$. These data also confirm the role of bradykin in in stimulating phosphatidylinositol bisphosphate hydrolys is as previously observed in radiolabeling studies.

In summary, a newly developed method for the measurement of inositol phosphate mass has been applied to distal renal epithelial cells. The present study demonstrates the utility of this methodology for measuring inositol phosphates in freshly isolated tissues and represents the first documentation of changes in inositol phosphate mass in non-transformed renal epithelia. Moreover, it demonstrates that kinin stimulation of renal papillary collecting tubule cells results in the formation of chemical levels of $I_{1}$ and $I_{3}$ which are consistent with the concurrent hydrolys is of phosphatidylinositol and phosphatidylinositol bisphosphate but not with hydrolysis of phosphatidylinositol bisphosphate alone. 


\section{ACKNOWLEDGMENT}

This work was supported by PHS award DK 01784-03.

\section{REFERENCES}

1. Shayman, J.A., Morrison, A.R. (1985) J. Clin. Invest. 76:978-984.

2. Shayman, J.A., Hruska, K.A., Morrison, A.R. (1986) Biochem. Biophys. Res. Corm. 134:299-304.

3. Shayman, J.A., Auchus, R.J., Morrison, A.R. (1986) Biochim. Biophys. Acta 888: 171-175.

4. Shayman, J.A., Morrison, A.R., Lowry, O.H. (1987) Anal. Bioch. (in press).

5. Undenfriend, S., Stein, S., Bohlen, P., Dairman, W., Leingruber, W., Weigele, M. (1972) Science 178:871-872.

6. MacGregor, L.G., Matschinsky, F.M. (1984) Anal. Biochem. 141:382-389.

7. Kato, T., Berger, S.J., Carter, J.A., Lowry, 0.H. (1973) Anai. Biochem. $53: 86-97$.

8. Rittenhouse, S., Sasson, J.P. (1985) J. Biol. Chem. 260:8657-8660.

9. Meek, J.L. (1986) Proc. Nat). Acad. Sci. 83:4162-4166.

10. Wilson, D.B., Neufeld, J., Majerus, P.W. (1985) J. Bio1. Chem. 260:1046-1051. 